\title{
Examining the Nature of Item Bias on Students' Performance in National Examinations Council (NECO) Mathematics Senior School Certificate Dichotomously Scored Items in Nigeria
}

\author{
A. Alaba Adediwura ${ }^{1}$, Asowo A. Patricia ${ }^{1}$ \\ ${ }^{1}$ Department of Educational Foundations and Counselling, Obafemi Awolowo University, Ile-Ife, Nigeria \\ Correspondence: Asowo A. Patricia, Department of Educational Foundations and Counselling, Obafemi Awolowo \\ University, Ile-Ife, Nigeria.
}

Received: August 10, 2020

Accepted: September 13, 2021

Online Published: November 7, 2021

doi:10.11114/ijce.v5i1.5402

URL: https://doi.org/10.11114/ijce.v5i1.5402

\begin{abstract}
This study examined the nature of item bias on students' performance in 2017 National Examinations Council (NECO) mathematics senior school certificate dichotomously scored items in Nigeria. The study adopted an ex-post-facto research design. A sample of 256,039 candidates was randomly selected from the population of 1,034,629 students who took the test. Instrument for data collection was 'Student Results' (SR). Data collected were analysed using the R language environment and an independent t-test. Results showed that the 2017 NECO Mathematics test was essentially unidimensional $(-0.28(<.20)$, ASSI $=-0.31(<0.25)$ and RATIO $=-0.31(<0.36)$. Results also showed that the nature of bias statistically encountered was a mean difference in scores bias, indicating that $86 \%$ ( 52 items), $79.1 \%$ (34 items), and 96\% (56 items) were biased against male students, urban and public-school students, respectively. It was concluded that item bias is a notable factor that affected the validity of the NECO 2017 Mathematics test and conclusions drawn from the scores in Nigeria. Hence, it was recommended that before tests are administered for public use, examination bodies should make a careful review of tests through dimensionality assessment at the developmental stage to eliminate any perspectives that could cause test inequity among examinees.
\end{abstract}

Keywords: schooling, national examinations, Item bias, Mental true test score approach

\section{Introduction}

Schooling as a tool is a channel through which formal education is achieved which was planned by society to help individuals reach self-actualisation. This is envisaged to attain development through investment in human capital formation to bridge the gap between different classes of people as a result of colour, country, technology, religion, school type, social values and beliefs, ethnicity and sex differences in the society. The aim of schooling in Nigeria, as stated in the National Policy on Education (FGN, 2013), is to prepare the individual child for a better existence in society. This is done by developing a national curriculum programme that would satisfy the basic needs required for higher education. It is at the end of their non-stop training years that public certificates' examinations that are a standard prerequisite for admission into tertiary institutions are taken.

\section{National Examinations}

In Nigeria, national examinations are done to select and place students in various educational institutions for the period of their training. Among these national examinations come the ones that are implemented during the transition from secondary education to tertiary education, such as NECO, WAEC and NABTEB; leaving candidates with the choice of either writing the 3 exams or certainly one of them. However, since the test is used for all involved groups from one state to the other every year, it is frequently simple to assume that the obtained results are comparable between groups. Besides, it can be remarked on whether the structures and contents of the items in the tests provide neither advantage nor disadvantage to any of the subgroups taking the exam. If the items in the tests provide an advantage for a group because of various features such as sex, school location, school type, race, social values and beliefs, ethnicity, or technology. It might be that the exam is biased in favour of that group which would negatively affect the validity of the selections made. So, a critical look at the perception of individuals on such standard examination in Nigeria might indicate a serious nature of item bias. 


\section{Item Bias}

Bandele and Aborisade (2018) explain that item bias affects the vital psychometric properties of measurements results in terms of validity and reliability. It occurs when there is a vast distinction in the performance between male and female students, private and public schools, schools in urban and rural areas, religious views, race, social values and beliefs or from state to state. Aborisade (2016) further explained that when students that have comparable ability levels are exposed to the same course content, they should have an 'equal probability of success' irrespective of the subgroup of the population to which they belong. The scholar maintained that if the examination items contain any source of difficulty that is not relevant to the construct being measured, these extraneous sources affect examinees performance. To be able to bridge the gap between them, examination agencies are expected to conduct tests that would reflect the true behaviour of individual examinees. However, if the validity and reliability of constructed items could not be ascertained before administration. Perhaps, some examination bodies do not include item bias detection in their item analysis.

\section{The Mental True Test Score Approach}

The Mental True Test Score approach (MTTSA) has been a modern-day technique that depicts a thorough picture of item functioning among examinees. It expects how passable an examinee would perform on a test. This framework proposes two distinctive units which, when jointly considered, are responsible for observing examinee response patterns in a standardised test. The first unit is the set of unobserved values of individual abilities, which each test taker possesses, denoted by $\theta$. This ability allows measurement experts to make a concomitant comparison of performance between examinees that have taken the test. The second unit is the set of responses essential at the item level. Such properties may reflect how difficult or extreme the items are. It would tell how well the items discriminate individuals along the scale, whether the probability of correct responses monotonically relates to individual ability and many more. However, with the MTST approach, the analysis of complex interaction patterns between subgroups, individual factors and item characteristics may detect biased items that are present in a multiple-choice test (Ahmad, Mokshein, and Husin, 2018).

In Nigeria, there have been a lot of studies on test/item bias that were both internally and internationally recognised. These studies have shown biased items across various dimensions of bias using more of the traditional differential item functioning approach and less of the modern mental true score approach to measuring irrelevant construct in a test. If examination agencies do not ensure those test items are bias-free using efficient effective methods, decisions made from the test items or test scores may overestimate or underestimate students' performance. If this happens, it would significantly affect the interpretation of conclusions made from scores which may deprive candidates who desire to study a hi-tech oriented course in tertiary institutions to lose admission or place them on courses they do not request (Orluwene \& Asiegbu, 2009; Nworgu, 2010; Adedoyin, 2010; Madu, 2011; Ogbebor and Onuka, (2013); Uremu \& Adams, 2013; Agbir, 2014, and Bandele \& Aborisade, 2018). It might also turn away the confidence parents and society have in the effectiveness of tests. Therefore, analysis of the probability of correct response for examinees and nature of bias encountered should be done to ascertain the reliability of the 2017 NECO SSCE mathematics multiple-choice test and possible conclusions drawn from the test scores in Nigeria.

\section{Purpose of the study}

The purpose of the study was to find out the nature of bias encountered on students' performance. The study, also, ascertains the dimensionality of the test and identified items that are biased based on the probability of correct responses of examinees from comparable ability levels using the MMTST approach.

\section{Research Questions}

i. How many dimensions have NECO 2017 Mathematics test among senior school students in Nigeria?

ii. What is the nature of bias statistically encountered in NECO SSCE 2017 dichotomously scored Mathematics items in Nigeria based on?
a. Sex?
b. School location?
c. School type?

\section{Method}

The study employed an Ex-post facto research design. The population consisted of all 1,034,629 students who sat for NECO SSCE 2017 Mathematics multiple-choice examination in Nigeria. A sample of 256,039 students representing all year three senior secondary (SSSIII) students that registered and sat for the examination in twelve states was selected using a non-proportional stratified random sampling technique with geopolitical zones serving as the basis for stratification. Samples were cluster-based on sex, school location, and school type for data analysis. The instrument for the study was “Student Results' (SR). Data collected were analysed using an R language environment. Supplementary 
item response theory (SIRT) was used to test dimensionality, Unidimensional item response theory (UMIRT) was used to estimate item parameters, factor analysis and t-test with the support of SPSS was performed to re-affirm test dimensionality and bias. Also, excel was used to estimates students' true scores.

\section{Results}

Research Question One: How many dimensions have NECO 2017 Mathematics test among senior school students in Nigeria? Here, the responses of examinees that sat for the 2017 National Examinations Council (NECO) mathematics test in Nigeria was subjected to Stout's test of essential unidimensionality and factor analysis.

Table 1. Dimensionality Assessment of 2017 NECO Mathematics dichotomously scored items in Nigeria under STEU

\begin{tabular}{lrr}
\hline & Unweighted & Weighted \\
\hline DETECT & -0.28 & -0.28 \\
ASSI & -0.31 & -0.31 \\
RATIO & -0.28 & -0.28
\end{tabular}

Table 1 showed that the assumption of unidimensionality was not violated. This is because the criteria of adjudging essential dimensionality of a test stand on the following basis $(0.20<$ DETECT $<1.00$, ASSI $<0.25$, Ratio $<0.36)$ according to Jang and Roussos, (2007) and Zhang (2007). This table showed that the 2017 Mathematics multiple-choice test was optimal and essentially unidimensional (maximum DETECT value $=-0.28(<.20)$, ASSI $=-0.31(<0.25)$ and RATIO $=-0.31(<0.36)$. The DETECT showed that the substantive test structure based on its purpose is consistent with the statistical dimensional structure at that level. Also, the ASSI and Ratio values revealed that examinee response data displayed an approximately simple structure. This implies that one dominant dimension accounted for the variation observed in student's responses to the mathematics dichotomously scored items in Nigeria. 
Table 2. Total Variance Explained

\begin{tabular}{|c|c|c|c|c|c|c|}
\hline \multirow[b]{2}{*}{ Component } & \multicolumn{3}{|c|}{ Initial Eigenvalues } & \multicolumn{3}{|c|}{ Extraction Sums of Squared Loadings } \\
\hline & Total & $\%$ of Variance & Cumulative \% & Total & $\%$ of Variance & Cumulative \% \\
\hline 1 & 11.618 & 19.363 & 19.363 & 11.618 & 19.363 & 19.363 \\
\hline 2 & 2.490 & 4.150 & 23.513 & 2.490 & 4.150 & 23.513 \\
\hline 3 & 2.386 & 3.977 & 27.489 & 2.386 & 3.977 & 27.489 \\
\hline 4 & 2.078 & 3.463 & 30.953 & 2.078 & 3.463 & 30.953 \\
\hline 5 & 1.948 & 3.246 & 34.199 & 1.948 & 3.246 & 34.199 \\
\hline 6 & 1.655 & 2.758 & 36.957 & 1.655 & 2.758 & 36.957 \\
\hline 7 & 1.277 & 2.128 & 39.085 & 1.277 & 2.128 & 39.085 \\
\hline 8 & 1.140 & 1.901 & 40.986 & 1.140 & 1.901 & 40.986 \\
\hline 9 & 1.085 & 1.809 & 42.795 & 1.085 & 1.809 & 42.795 \\
\hline 10 & 1.060 & 1.767 & 44.562 & 1.060 & 1.767 & 44.562 \\
\hline 11 & 1.015 & 1.691 & 46.253 & 1.015 & 1.691 & 46.253 \\
\hline 12 & .988 & 1.646 & 47.899 & & & \\
\hline 13 & .965 & 1.608 & 49.508 & & & \\
\hline 14 & .935 & 1.558 & 51.066 & & & \\
\hline 15 & .890 & 1.483 & 52.549 & & & \\
\hline 16 & .869 & 1.448 & 53.996 & & & \\
\hline 17 & .833 & 1.389 & 55.385 & & & \\
\hline 18 & .827 & 1.378 & 56.763 & & & \\
\hline 19 & .795 & 1.325 & 58.088 & & & \\
\hline 20 & .787 & 1.312 & 59.400 & & & \\
\hline 21 & .773 & 1.288 & 60.688 & & & \\
\hline 22 & .770 & 1.284 & 61.972 & & & \\
\hline 23 & .766 & 1.277 & 63.249 & & & \\
\hline 24 & .748 & 1.247 & 64.496 & & & \\
\hline 25 & .737 & 1.228 & 65.724 & & & \\
\hline 26 & .723 & 1.205 & 66.928 & & & \\
\hline 27 & .715 & 1.192 & 68.121 & & & \\
\hline 28 & .706 & 1.176 & 69.297 & & & \\
\hline 29 & .698 & 1.163 & 70.460 & & & \\
\hline 30 & .689 & 1.148 & 71.609 & & & \\
\hline 31 & .677 & 1.128 & 72.737 & & & \\
\hline 32 & .672 & 1.121 & 73.857 & & & \\
\hline 33 & .667 & 1.111 & 74.968 & & & \\
\hline 34 & .660 & 1.100 & 76.068 & & & \\
\hline 35 & .647 & 1.078 & 77.146 & & & \\
\hline 36 & .643 & 1.071 & 78.217 & & & \\
\hline 37 & .631 & 1.052 & 79.270 & & & \\
\hline 38 & .623 & 1.038 & 80.308 & & & \\
\hline 39 & .612 & 1.020 & 81.327 & & & \\
\hline 40 & .606 & 1.010 & 82.337 & & & \\
\hline 41 & .604 & 1.006 & 83.343 & & & \\
\hline 42 & .592 & .987 & 84.331 & & & \\
\hline 43 & .586 & .977 & 85.308 & & & \\
\hline 44 & .574 & .956 & 86.264 & & & \\
\hline 45 & .570 & .951 & 87.215 & & & \\
\hline 46 & .561 & .935 & 88.150 & & & \\
\hline 47 & .554 & .924 & 89.073 & & & \\
\hline 48 & .551 & .919 & 89.992 & & & \\
\hline 49 & .537 & .896 & 90.888 & & & \\
\hline 50 & .534 & .889 & 91.777 & & & \\
\hline 51 & .527 & .878 & 92.655 & & & \\
\hline 52 & .519 & .865 & 93.521 & & & \\
\hline 53 & .515 & .859 & 94.380 & & & \\
\hline 54 & .506 & .844 & 95.223 & & & \\
\hline 55 & .501 & .836 & 96.059 & & & \\
\hline 56 & .493 & .822 & 96.881 & & & \\
\hline 57 & .488 & .814 & 97.695 & & & \\
\hline 58 & .478 & .796 & 98.492 & & & \\
\hline 59 & .464 & .773 & 99.265 & & & \\
\hline 60 & .441 & .735 & 100.000 & & & \\
\hline
\end{tabular}

Table 2 showed the extraction from the principal component analysis after interacting communalities revealed 11 components with eigenvalues greater than 1 . This explained 19.363, 4.150, 3.977, 3.463, 3.246, 2.758, 2.128, 1.901, 
$1.809,1.767$, and $1.691 \%$ of variance accounted for by each component to the total variance in all the scores. For the scores from the 60 multiple-choice Mathematics items, regarding the eigenvalues greater than 1, the total percentage variance was 46.253. from the results of PCA, the items were unidimensional because the first factor (19.363) extracted exceeded the second factor (4.751) by a reasonable distance. In addition, a scree plot was used to confirm the dimensionality of the test.

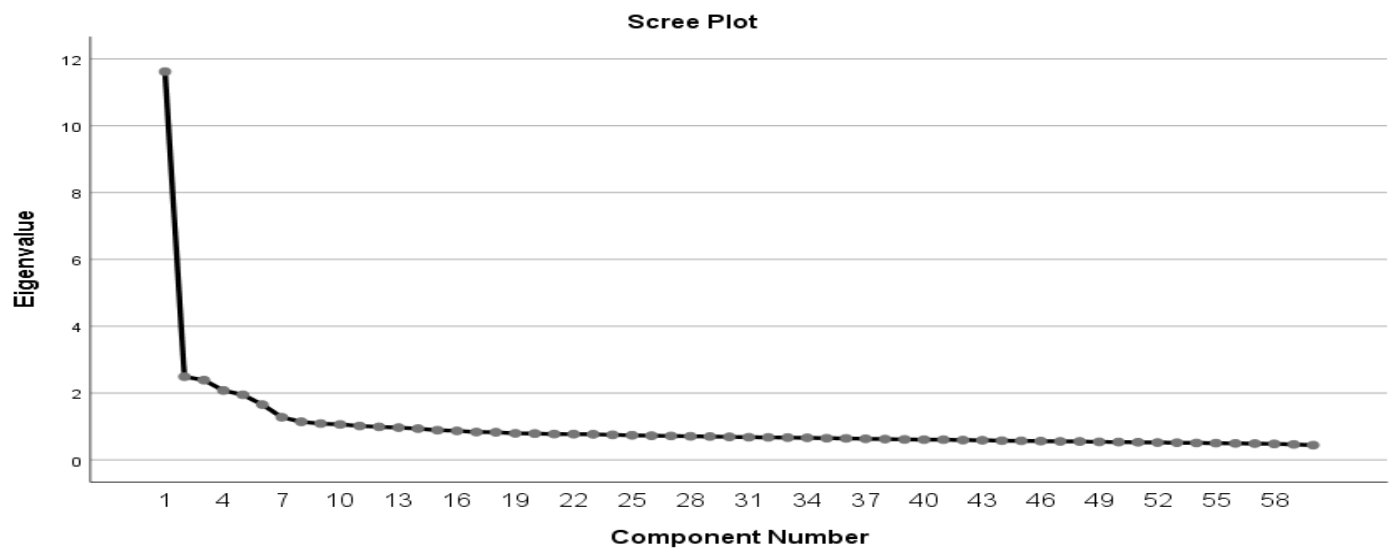

Figure 1. Scree Plot of Examinee Scores in the 2017 NECO Mathematics items

Results from the scree plot showed a visual of how the total variance associated with each factor. The steep slope revealed the largest factors associated with the loading greater than the eigenvalues of 1 . Similarly, the gradual trailing off-screen showed the rest of the factors lower than eigenvalues of 1 . Therefore, one distinct factor with larger eigenvalues in each case implies that the items were unidimensional.

Research Question Two: What is the nature of bias statistically encountered in 2017 NECO SSC dichotomously scored Mathematics items in Nigeria based on sex, school location and school type? Here, four levels of preliminary analyses were conducted before tentatively determining statistically the nature of bias encountered using MMTTST procedures.,

The first is DIF analysis. Here, we subject the responses of examinees from the 2017 SSC NECO Mathematics test to a Unidimensional differential item functioning (UDIF) investigation implemented in the DIF package of $\mathrm{R}$ language. The results revealed that 52,43 , and 58 of the items showed an incidence of DIF at 0.05 level of significance concerning sex, school location and school type. This implies that $86 \%, 71 \%$ and $96 \%$ of the 2017 NECO Mathematics multiple-choice test items functioned differently for candidates based on their sex, school location and school type (see appendices 1,2 $\& 3$ ). Second, examinee scores were subjected to item calibration of the $\mathrm{R}$ language for ability estimates and model fit. Thereafter, SPSS was used to group the ability estimates to identify examinees that have the same ability estimates. The result showed an ability estimate of 0.2 as the ability estimate with the highest frequency $(13,274)$ as a base for the identification of candidates that have the same ability estimate. The result implies that the examinee that falls into the same ability group had the same probability of answering the items correctly which may be an indication of administrative errors on the part of the examination agency, cheating or examination malpractice among the students (see appendix 4). Third, the probability of correct responses for all candidates with the same ability $(13,274)$ scores along their groups was calculated using the three-parameter logistic model that fitted the data. From the abridged table concerning sex, school location and school type, the results revealed that there were too many high scores among the candidates on many items consecutively (see appendices 4). This might imply having too many easy items on the test, the presence of cheating among students or administrative error before test administration. Finally, the probability of examinee correct responses was subjected to a comparative estimation to examine the nature of bias encountered statistically along with groups. 
Table 3. Set of DIF Items that Tentatively Showed the Nature of Bias Encountered Statistically Based on Sex

\begin{tabular}{|c|c|c|c|c|c|c|c|c|c|c|}
\hline Items & Sex & $\mathbf{N}$ & Mean & $\begin{array}{c}\text { Std. } \\
\text { Deviation }\end{array}$ & Mean Diff & $\mathbf{t}$ & df & $\begin{array}{c}\text { Sig. } \\
(2-\text {-tailed })\end{array}$ & Remark & Evaluation \\
\hline \multirow[t]{2}{*}{ IT1 } & Male & 6973 & .7889 & .17160 & -.04854 & -17.758 & 13272 & .000 & Sig & Biased against male \\
\hline & Female & 6301 & .8374 & .13966 & & & & & & \\
\hline \multirow[t]{2}{*}{ IT3 } & Male & 6973 & .8040 & .05133 & -.01075 & -11.401 & 13272 & .000 & Sig & Biased against male \\
\hline & Female & 6301 & .8148 & .05726 & & & & & & \\
\hline \multirow[t]{2}{*}{ IT4 } & Male & 6973 & .7627 & .23958 & -.05047 & -13.115 & 13272 & .000 & Sig & Biased against male \\
\hline & Female & 6301 & .8131 & .19940 & & & & & & \\
\hline \multirow[t]{2}{*}{ IT5 } & Male & 6973 & .6438 & .00911 & -.03179 & -142.923 & 13272 & .000 & Sig & Biased against male \\
\hline & Female & 6301 & .6756 & .01591 & & & & & & \\
\hline \multirow[t]{2}{*}{ IT7 } & Male & 6973 & .7181 & .25581 & -.06612 & -15.927 & 13272 & .000 & Sig & Biased against male \\
\hline & Female & 6301 & .7842 & .21848 & & & & & & \\
\hline \multirow[t]{2}{*}{ IT8 } & Male & 6973 & .8150 & .14351 & -.02486 & -10.317 & 13272 & .000 & Sig & Biased against male \\
\hline & Female & 6301 & .8398 & .13298 & & & & & & \\
\hline \multirow[t]{2}{*}{ IT9 } & Male & 6973 & .6558 & .26571 & -.09243 & -21.123 & 13272 & .000 & Sig & Biased against male \\
\hline & Female & 6301 & .7483 & .23533 & & & & & & \\
\hline \multirow[t]{2}{*}{ IT10 } & Male & 6973 & .7074 & .17795 & -.04428 & -14.762 & 13272 & .000 & Sig & Biased against male \\
\hline & Female & 6301 & .7517 & .16639 & & & & & & \\
\hline \multirow[t]{2}{*}{ IT11 } & Male & 6973 & .6666 & .29153 & -.07655 & -16.152 & 13272 & .000 & Sig & Biased against male \\
\hline & Female & 6301 & .7431 & .25016 & & & & & & \\
\hline \multirow{2}{*}{ IT12 } & Male & 6973 & .5831 & .26520 & -.07978 & -18.441 & 13272 & .000 & Sig & Biased against male \\
\hline & Female & 6301 & .6629 & .22948 & & & & & & \\
\hline \multirow[t]{2}{*}{ IT13 } & Male & 6973 & .0700 & .00238 & -.00436 & -31.594 & 13272 & .000 & Sig & Biased against male \\
\hline & Female & 6301 & .0744 & .01126 & & & & & & \\
\hline \multirow[t]{2}{*}{ IT15 } & Male & 6973 & .5706 & .24527 & -.07695 & -19.068 & 13272 & .000 & Sig & Biased against male \\
\hline & Female & 6301 & .6476 & .21679 & & & & & & \\
\hline \multirow[t]{2}{*}{ IT16 } & Male & 6973 & .7378 & .16926 & -.05900 & -20.299 & 13272 & .000 & Sig & Biased against male \\
\hline & Female & 6301 & .7968 & .16492 & & & & & & \\
\hline \multirow[t]{2}{*}{ IT17 } & Male & 6973 & .7221 & .26524 & -.07642 & -17.734 & 13272 & .000 & Sig & Biased against male \\
\hline & Female & 6301 & .7985 & .22722 & & & & & & \\
\hline IT19 & Male & 6973 & .8822 & .08559 & -.02469 & -17.283 & 13272 & .000 & Sig & Biased against male \\
\hline & Female & 6301 & .9069 & .07823 & & & & & & \\
\hline IT20 & Male & 6973 & .7450 & .18965 & -.05559 & -17.712 & 13272 & .000 & Sig & Biased against male \\
\hline & Female & 6301 & .8006 & .16999 & & & & & & \\
\hline IT21 & Male & 6973 & .4006 & .12118 & -.03117 & -15.778 & 13272 & .000 & Sig & Biased against male \\
\hline & Female & 6301 & .4318 & .10472 & & & & & & \\
\hline IT22 & Male & 6973 & .7430 & .25683 & -.07440 & -18.458 & 13272 & .000 & Sig & Biased against male \\
\hline & Female & 6301 & .8175 & .20078 & & & & & & \\
\hline IT23 & Male & 6973 & .5171 & .06377 & -.02852 & -23.760 & 13272 & .000 & Sig & Biased against male \\
\hline & Female & 6301 & .5456 & .07445 & & & & & & \\
\hline IT25 & Male & 6973 & .4935 & .00085 & -.02036 & -1903.144 & 13272 & .000 & Sig & Biased against male \\
\hline & Female & 6301 & .5138 & .00007 & & & & & & \\
\hline IT26 & Male & 6973 & .7536 & .21139 & -.05550 & -16.969 & 13272 & .000 & Sig & Biased against male \\
\hline & Female & 6301 & .8091 & .15853 & & & & & & \\
\hline IT27 & Male & 6973 & .5628 & .21101 & -.06929 & -20.362 & 13272 & .000 & Sig & Biased against male \\
\hline & Female & 6301 & .6321 & .17739 & & & & & & \\
\hline IT29 & Male & 6973 & .3620 & .11580 & -.03581 & -19.420 & 13272 & .000 & Sig & Biased against male \\
\hline & Female & 6301 & .3978 & .09421 & & & & & & \\
\hline IT30 & Male & 6973 & .7553 & .13798 & -.05050 & -21.562 & 13272 & .000 & Sig & Biased against male \\
\hline & Female & 6301 & .8058 & .13106 & & & & & & \\
\hline IT31 & Male & 6973 & .6423 & .28756 & -.07972 & -17.131 & 13272 & .000 & Sig & Biased against male \\
\hline & Female & 6301 & .7220 & .24393 & & & & & & \\
\hline IT32 & Male & 6973 & .6804 & .30485 & -.08710 & -17.756 & 13272 & .000 & Sig & Biased against male \\
\hline & Female & 6301 & .7675 & .25481 & & & & & & \\
\hline IT33 & Male & 6973 & .6792 & .27797 & -.08287 & -18.351 & 13272 & .000 & Sig & Biased against male \\
\hline & Female & 6301 & .7621 & .23813 & & & & & & \\
\hline IT34 & Male & 6973 & .7076 & .28808 & -.07834 & -16.982 & 13272 & .000 & Sig & Biased against male \\
\hline & Female & 6301 & .7859 & .23776 & & & & & & \\
\hline IT35 & Male & 6973 & .7264 & .27563 & -.08066 & -18.234 & 13272 & .000 & Sig & Biased against male \\
\hline & Female & 6301 & .8071 & .22882 & & & & & & \\
\hline
\end{tabular}




\begin{tabular}{|c|c|c|c|c|c|c|c|c|c|c|}
\hline \multirow[t]{2}{*}{ IT36 } & Male & 6973 & .5755 & .27042 & -.08760 & -19.840 & 13272 & .000 & Sig & Biased against male \\
\hline & Female & 6301 & .6631 & .23451 & & & & & & \\
\hline \multirow[t]{2}{*}{ IT37 } & Male & 6973 & .5354 & .26733 & -.07733 & -17.112 & 13272 & .000 & Sig & Biased against male \\
\hline & Female & 6301 & .6127 & .25164 & & & & & & \\
\hline \multirow[t]{2}{*}{ IT38 } & Male & 6973 & .6976 & .20329 & -.06318 & -18.249 & 13272 & .000 & Sig & Biased against male \\
\hline & Female & 6301 & .7608 & .19457 & & & & & & \\
\hline \multirow[t]{2}{*}{ IT40 } & Male & 6973 & .5831 & .26847 & -.07183 & -16.274 & 13272 & .000 & Sig & Biased against male \\
\hline & Female & 6301 & .6549 & .23681 & & & & & & \\
\hline \multirow[t]{2}{*}{ IT41 } & Male & 6973 & .6754 & .19261 & -.05174 & -15.990 & 13272 & .000 & Sig & Biased against male \\
\hline & Female & 6301 & .7271 & .17880 & & & & & & \\
\hline \multirow[t]{2}{*}{ IT42 } & Male & 6973 & .5072 & .25361 & -.08275 & -19.414 & 13272 & .000 & Sig & Biased against male \\
\hline & Female & 6301 & .5900 & .23558 & & & & & & \\
\hline \multirow[t]{2}{*}{ IT43 } & Male & 6973 & .6925 & .26461 & -.07589 & -17.730 & 13272 & .000 & Sig & Biased against male \\
\hline & Female & 6301 & .7684 & .22418 & & & & & & \\
\hline \multirow[t]{2}{*}{ IT44 } & Male & 6973 & .5591 & .06170 & -.03920 & -35.230 & 13272 & .000 & Sig & Biased against male \\
\hline & Female & 6301 & .5983 & .06649 & & & & & & \\
\hline \multirow[t]{2}{*}{ IT45 } & Male & 6973 & .7114 & .21861 & -.06417 & -17.319 & 13272 & .000 & Sig & Biased against male \\
\hline & Female & 6301 & .7755 & .20695 & & & & & & \\
\hline \multirow[t]{2}{*}{ IT46 } & Male & 6973 & .7653 & .25862 & -.07702 & -18.785 & 13272 & .000 & Sig & Biased against male \\
\hline & Female & 6301 & .8423 & .20784 & & & & & & \\
\hline \multirow[t]{2}{*}{ IT47 } & Male & 6973 & .5548 & .16748 & -.06063 & -22.209 & 13272 & .000 & Sig & Biased against male \\
\hline & Female & 6301 & .6155 & .14467 & & & & & & \\
\hline \multirow[t]{2}{*}{ IT48 } & Male & 6973 & .6954 & .20393 & -.06106 & -17.566 & 13272 & .000 & Sig & Biased against male \\
\hline & Female & 6301 & .7565 & .19550 & & & & & & \\
\hline \multirow[t]{2}{*}{ IT49 } & Male & 6973 & .7706 & .16609 & -.05866 & -21.693 & 13272 & .000 & Sig & Biased against male \\
\hline & Female & 6301 & .8292 & .14305 & & & & & & \\
\hline \multirow[t]{2}{*}{ IT50 } & Male & 6973 & .6711 & .18821 & -.04565 & -14.366 & 13272 & .000 & Sig & Biased against male \\
\hline & Female & 6301 & .7167 & .17670 & & & & & & \\
\hline \multirow[t]{2}{*}{ IT51 } & Male & 6973 & .8212 & .16457 & -.04041 & -15.924 & 13272 & .000 & Sig & Biased against male \\
\hline & Female & 6301 & .8616 & .12217 & & & & & & \\
\hline \multirow[t]{2}{*}{ IT52 } & Male & 6973 & .8296 & .19878 & -.04918 & -15.436 & 13272 & .000 & Sig & Biased against male \\
\hline & Female & 6301 & .8788 & .16452 & & & & & & \\
\hline \multirow[t]{2}{*}{ IT53 } & Male & 6973 & .7725 & .14983 & -.04283 & -16.555 & 13272 & .000 & Sig & Biased against male \\
\hline & Female & 6301 & .8153 & .14775 & & & & & & \\
\hline \multirow[t]{2}{*}{ IT54 } & Male & 6973 & .7669 & .21608 & -.05275 & -14.748 & 13272 & .000 & Sig & Biased against male \\
\hline & Female & 6301 & .8196 & .19373 & & & & & & \\
\hline \multirow[t]{2}{*}{ IT55 } & Male & 6973 & .3592 & .07253 & -.04667 & -36.575 & 13272 & .000 & Sig & Biased against male \\
\hline & Female & 6301 & .4059 & .07439 & & & & & & \\
\hline \multirow[t]{2}{*}{ IT56 } & Male & 6973 & .7175 & .15271 & -.03217 & -12.089 & 13272 & .000 & Sig & Biased against male \\
\hline & Female & 6301 & .7496 & .15352 & & & & & & \\
\hline \multirow[t]{2}{*}{ IT57 } & Male & 6973 & .7393 & .21689 & -.06091 & -17.116 & 13272 & .000 & Sig & Biased against male \\
\hline & Female & 6301 & .8002 & .19042 & & & & & & \\
\hline IT59 & Male & 6973 & .6598 & .23601 & -.05954 & -15.205 & 13272 & .000 & Sig & Biased against male \\
\hline & Female & 6301 & .7193 & .21277 & & & & & & \\
\hline IT60 & Male & 6973 & .5038 & .12499 & -.03756 & -18.303 & 13272 & .000 & Sig & Biased against male \\
\hline & Female & 6301 & .5414 & .10986 & & & & & & \\
\hline
\end{tabular}

Significant at $\mathrm{p} \leq 0.05$

Table 3 showed the disparity between individual examinee performances on the item with mean score differences at face value. The table revealed that ( $86 \%$ of items) 52 scores $(1,3,4,8,9,10,11,12,13,15,16,17,19,20,21,22,23$, $25,26,27,29,30,31,32,33,34,35,36,37,40,45,46,47,48,49,50,51,52,53,54,55,56,59$ and 60 ) were biased against male students aside eight items with logits greater than .05. Likewise, female scores showed they had the skills needed to answer difficult items with possible higher computation skills and on easier items that require basic applications. From the scores, the number of significant items by group interaction upon the assumption that there was an equivalent distribution of abilities across groups. It specified that the test is measuring irrelevant factors that are no longer related to the construct being assessed. This implies that the mean difference in score bias has contributed largely to the non-equivalency of ability distribution among males and females. This might have affected the construct and content validity of the test. 
Table 4. Set of DIF Items that tentatively showed the Nature of Bias encountered statistically based on School Location

\begin{tabular}{|c|c|c|c|c|c|c|c|c|c|c|}
\hline & Location & $\mathbf{N}$ & Mean & $\begin{array}{c}\text { Std. } \\
\text { Deviation }\end{array}$ & $\begin{array}{l}\text { Mean } \\
\text { Diff }\end{array}$ & t & df & $\begin{array}{c}\text { Sig. } \\
\text { (2-tailed) }\end{array}$ & Remark & Evaluation \\
\hline \multirow[t]{2}{*}{ IT1 } & Rural & 4728 & .8416 & .11844 & .04703 & 16.327 & 13272 & .000 & sig & Biased against Urban \\
\hline & Urban & 8546 & .7946 & .17739 & & & & & & \\
\hline \multirow[t]{2}{*}{ IT2 } & Rural & 4728 & .5420 & .04960 & .03431 & 37.919 & 13272 & .000 & sig & Biased against Urban \\
\hline & Urban & 8546 & .5077 & .05009 & & & & & & \\
\hline \multirow[t]{2}{*}{ IT4 } & Rural & 4728 & .8244 & .17840 & .05812 & 14.500 & 13272 & .000 & sig & Biased against Urban \\
\hline & Urban & 8546 & .7662 & .24157 & & & & & & \\
\hline \multirow[t]{2}{*}{ IT5 } & Rural & 4728 & .6465 & .00311 & -.01671 & -56.375 & 13272 & .000 & sig & Biased against Rural \\
\hline & Urban & 8546 & .6632 & .02025 & & & & & & \\
\hline \multirow[t]{2}{*}{ IT6 } & Rural & 4728 & .8302 & .06813 & .00896 & 5.874 & 13272 & .000 & sig & Biased against Urban \\
\hline & Urban & 8546 & .8213 & .09181 & & & & & & \\
\hline \multirow[t]{2}{*}{ IT7 } & Rural & 4728 & .7906 & .19437 & .06302 & 14.560 & 13272 & .000 & sig & Biased against Urban \\
\hline & Urban & 8546 & .7275 & .26015 & & & & & & \\
\hline \multirow[t]{2}{*}{ IT8 } & Rural & 4728 & .8533 & .11870 & .04134 & 16.450 & 13272 & .000 & sig & Biased against Urban \\
\hline & Urban & 8546 & .8120 & .14852 & & & & & & \\
\hline \multirow[t]{2}{*}{ IT9 } & Rural & 4728 & .7490 & .22571 & .07931 & 17.442 & 13272 & .000 & sig & Biased against Urban \\
\hline & Urban & 8546 & .6697 & .26377 & & & & & & \\
\hline \multirow[t]{2}{*}{ IT10 } & Rural & 4728 & .7570 & .14428 & .04744 & 14.706 & 13272 & .000 & sig & Biased against Urban \\
\hline & Urban & 8546 & .7095 & .19413 & & & & & & \\
\hline \multirow[t]{2}{*}{ IT11 } & Rural & 4728 & .7378 & .23349 & .05373 & 10.800 & 13272 & .000 & sig & Biased against Urban \\
\hline & Urban & 8546 & .6841 & .29475 & & & & & & \\
\hline \multirow{2}{*}{ IT12 } & Rural & 4728 & .6669 & .22042 & .07276 & 16.064 & 13272 & .000 & sig & Biased against Urban \\
\hline & Urban & 8546 & .5941 & .26479 & & & & & & \\
\hline IT13 & Rural & 4728 & .0706 & .00294 & -.00249 & -20.377 & 13272 & .000 & sig & Biased against Rural \\
\hline & Urban & 8546 & .0731 & .00812 & & & & & & \\
\hline IT14 & Rural & 4728 & .6991 & .23345 & .07030 & 15.087 & 13272 & .000 & sig & Biased against Urban \\
\hline & Urban & 8546 & .6288 & .26926 & & & & & & \\
\hline IT15 & Rural & 4728 & .6200 & .21639 & .01720 & 4.071 & 13272 & .000 & sig & Biased against Urban \\
\hline & Urban & 8546 & .6028 & .24182 & & & & & & \\
\hline IT16 & Rural & 4728 & .7973 & .14212 & .05384 & 17.343 & 13272 & .000 & sig & Biased against Urban \\
\hline & Urban & 8546 & .7435 & .18545 & & & & & & \\
\hline IT17 & Rural & 4728 & .7987 & .21067 & .06288 & 14.008 & 13272 & .000 & sig & Biased against Urban \\
\hline & Urban & 8546 & .7358 & .26595 & & & & & & \\
\hline IT18 & Rural & 4728 & .4909 & .09369 & -.00914 & -5.001 & 13272 & .000 & sig & Biased against Rural \\
\hline & Urban & 8546 & .5000 & .10462 & & & & & & \\
\hline IT20 & Rural & 4728 & .8046 & .14861 & .05270 & 16.001 & 13272 & .000 & sig & Biased against Urban \\
\hline & Urban & 8546 & .7519 & .19767 & & & & & & \\
\hline IT21 & Rural & 4728 & .4261 & .09778 & .01517 & 7.222 & 13272 & .000 & sig & Biased against Urban \\
\hline & Urban & 8546 & .4109 & .12482 & & & & & & \\
\hline IT24 & Rural & 4728 & .7797 & .16207 & .04649 & 13.636 & 13272 & .000 & sig & Biased against Urban \\
\hline & Urban & 8546 & .7332 & .20103 & & & & & & \\
\hline IT25 & Rural & 4728 & .4833 & .00106 & -.03154 & -933.288 & 13272 & .000 & sig & Biased against Rural \\
\hline & Urban & 8546 & .5149 & .00218 & & & & & & \\
\hline IT27 & Rural & 4728 & .6127 & .17218 & .02368 & 6.611 & 13272 & .000 & sig & Biased against Urban \\
\hline & Urban & 8546 & .5890 & .21043 & & & & & & \\
\hline IT28 & Rural & 4728 & .3859 & .06813 & .04900 & 26.121 & 13272 & .000 & sig & Biased against Urban \\
\hline & Urban & 8546 & .3369 & .11861 & & & & & & \\
\hline IT29 & Rural & 4728 & .3637 & .10255 & -.03045 & -15.696 & 13272 & .000 & sig & Biased against Rural \\
\hline & Urban & 8546 & .3941 & .10941 & & & & & & \\
\hline IT30 & Rural & 4728 & .7864 & .11371 & .01220 & 4.817 & 13272 & .000 & sig & Biased against Urban \\
\hline & Urban & 8546 & .7742 & .15230 & & & & & & \\
\hline IT34 & Rural & 4728 & .7776 & .23588 & .04844 & 10.075 & 13272 & .000 & sig & Biased against Urban \\
\hline & Urban & 8546 & .7292 & .28018 & & & & & & \\
\hline IT37 & Rural & 4728 & .5773 & .25083 & .00713 & 1.497 & 13272 & .134 & NS & IMPACT \\
\hline & Urban & 8546 & .5702 & .26880 & & & & & & \\
\hline IT39 & Rural & 4728 & .7834 & .16479 & .19270 & 40.934 & 13272 & .000 & sig & Biased against Urban \\
\hline & Urban & 8546 & .5907 & .29959 & & & & & & \\
\hline IT40 & Rural & 4728 & .6357 & .23626 & -.00633 & -1.453 & 13272 & .146 & Sig & Biased against Rural \\
\hline & Urban & 8546 & .6420 & .24274 & & & & & & \\
\hline IT41 & Rural & 4728 & .7166 & .17926 & .18963 & 40.159 & 13272 & .000 & sig & Biased against Urban \\
\hline & Urban & 8546 & .5270 & .29604 & & & & & & \\
\hline IT44 & Rural & 4728 & .5754 & .04158 & -.03258 & -28.092 & 13272 & .000 & sig & Biased against Rural \\
\hline & Urban & 8546 & .6080 & .07351 & & & & & & \\
\hline IT45 & Rural & 4728 & .7678 & .18773 & .04253 & 10.782 & 13272 & .000 & sig & Biased against Urban \\
\hline & Urban & 8546 & .7252 & .23251 & & & & & & \\
\hline
\end{tabular}




\begin{tabular}{|c|c|c|c|c|c|c|c|c|c|c|}
\hline \multirow[t]{2}{*}{ IT46 } & Rural & 4728 & .8405 & .17838 & .04378 & 10.785 & 13272 & .000 & sig & Biased against Urban \\
\hline & Urban & 8546 & .7967 & .24557 & & & & & & \\
\hline \multirow[t]{2}{*}{ IT48 } & Rural & 4728 & .7599 & .17411 & .17765 & 36.903 & 13272 & .000 & sig & Biased against Urban \\
\hline & Urban & 8546 & .5823 & .30462 & & & & & & \\
\hline \multirow[t]{2}{*}{ IT50 } & Rural & 4728 & .7289 & .15687 & .08887 & 24.564 & 13272 & .000 & sig & Biased against Urban \\
\hline & Urban & 8546 & .6400 & .21970 & & & & & & \\
\hline \multirow[t]{2}{*}{ IT52 } & Rural & 4728 & .8785 & .12888 & .02765 & 8.715 & 13272 & .000 & sig & Biased against Urban \\
\hline & Urban & 8546 & .8509 & 19597 & & & & & & \\
\hline \multirow[t]{2}{*}{ IT53 } & Rural & 4728 & .7995 & .13858 & .21056 & 44.692 & 13272 & .000 & sig & Biased against Urban \\
\hline & Urban & 8546 & .5890 & .30712 & & & & & & \\
\hline \multirow[t]{2}{*}{ IT54 } & Rural & 4728 & .8237 & .15359 & .03370 & 9.492 & 13272 & .000 & sig & Biased against Urban \\
\hline & Urban & 8546 & .7900 & .21577 & & & & & & \\
\hline \multirow[t]{2}{*}{ IT55 } & Rural & 4728 & .3760 & .05352 & -.05535 & -41.773 & 13272 & .000 & sig & Biased against Urban \\
\hline & Urban & 8546 & .4314 & .08195 & & & & & & \\
\hline \multirow[t]{2}{*}{ IT56 } & Rural & 4728 & .7487 & .14412 & .22139 & 51.870 & 13272 & .000 & sig & Biased against Urban \\
\hline & Urban & 8546 & .5273 & .27321 & & & & & & \\
\hline \multirow[t]{2}{*}{ IT58 } & Rural & 4728 & .7998 & .14630 & .03409 & 9.878 & 13272 & .000 & sig & Biased against Urban \\
\hline & Urban & 8546 & .7657 & .21088 & & & & & & \\
\hline \multirow[t]{2}{*}{ IT59 } & Rural & 4728 & .7132 & .18624 & .01670 & 4.239 & 13272 & .000 & sig & Biased against Urban \\
\hline & Urban & 8546 & .6965 & .23281 & & & & & & \\
\hline \multirow[t]{2}{*}{ IT60 } & Rural & 4728 & .5287 & .10131 & -.02926 & -14.343 & 13272 & .000 & sig & Biased against Rural \\
\hline & Urban & 8546 & .5579 & .11830 & & & & & & \\
\hline
\end{tabular}

Significant at $\mathrm{p} \leq 0.05$

Table 4 illustrates the disparity between individual examinee performances on the item with mean score differences at face value among the comparison groups. The table showed that ( $79.1 \%$ of items) 34 scores $(1,2,4,6,7,7,8,10,11$, $12,14,15,16,17,20,24,27,28,30,34,39,41,45,46,47,48,50,52,53,54,55,56,58$ and 59) were biased against students from urban schools. Similarly, (18.6\% of items) 8 scores $(5,13,18,25,29,44$ and 60$)$ were biased against candidates from rural schools. It also revealed that score 37 (2.3\% of items) showed item impact, which means that the item measured the intended construct of the test. the results revealed that scores of students from urban areas showed they were at a disadvantage on difficult items that require higher calculation skills but were on an advantage at easier items that require the application of one to more simple basic mathematical operations based on the location of their schools. Likewise, students' scores from rural areas showed it advantaged them on difficult items that require higher calculation skills but maybe careless at easier items that require simple mathematical skills. It is possible from the score that candidates from urban schools lack good mastery of subject content and confident skills when the test was administered, which had put them in a disadvantaged position. The result further revealed that the relative difficulty of scores on the test based on school location has not remained constant considering the number of significant scores of group interaction. From the scores, it is unlikely that it met the assumption of the equivalent distribution of abilities across groups. The result implies that the nature of bias encountered based on school location was a mean difference in scores bias. Thus, a reflection of differential construct and content validity biases might have occurred along with the groups.

Table 4.1.2.3. Set of DIF Items that tentatively showed the Nature of Bias encountered statistically Based on School Type

\begin{tabular}{|c|c|c|c|c|c|c|c|c|c|c|}
\hline & Type & $\mathbf{N}$ & Mean & $\begin{array}{c}\text { Std. } \\
\text { Deviation }\end{array}$ & $\begin{array}{c}\text { Mean } \\
\text { Diff }\end{array}$ & $\mathbf{t}$ & df & $\begin{array}{c}\text { Sig. } \\
\text { (2-tailed) }\end{array}$ & Remark & Evaluation \\
\hline \multirow[t]{2}{*}{ IT1 } & Private & 6079 & .8395 & .12203 & .04590 & 17.404 & 13272 & .000 & Sig & Biased against public \\
\hline & Public & 7195 & .7936 & .17233 & & & & & & \\
\hline \multirow[t]{2}{*}{ IT2 } & Private & 6079 & .5421 & .07067 & .03151 & 31.794 & 13272 & .000 & Sig & Biased against public \\
\hline & Public & 7195 & .5105 & .04184 & & & & & & \\
\hline \multirow[t]{2}{*}{ IT3 } & Private & 6079 & .8349 & .06439 & .03863 & 39.473 & 13272 & .000 & Sig & Biased against public \\
\hline & Public & 7195 & .7963 & .04817 & & & & & & \\
\hline \multirow[t]{2}{*}{ IT4 } & Private & 6079 & .8264 & .16896 & .06673 & 17.914 & 13272 & .000 & Sig & Biased against public \\
\hline & Public & 7195 & .7597 & .24540 & & & & & & \\
\hline \multirow[t]{2}{*}{ IT5 } & Private & 6079 & .7283 & .05439 & .10294 & 158.369 & 13272 & .000 & Sig & Biased against public \\
\hline & Public & 7195 & .6254 & .00828 & & & & & & \\
\hline \multirow[t]{2}{*}{ IT6 } & Private & 6079 & .8382 & .06654 & .02342 & 16.679 & 13272 & .000 & Sig & Biased against public \\
\hline & Public & 7195 & .8148 & .09078 & & & & & & \\
\hline \multirow[t]{2}{*}{ IT8 } & Private & 6079 & .8442 & .13890 & .03120 & 12.756 & 13272 & .000 & Sig & Biased against public \\
\hline & Public & 7195 & .8130 & .14165 & & & & & & \\
\hline \multirow[t]{2}{*}{ IT9 } & Private & 6079 & .7246 & .21965 & .04705 & 10.800 & 13272 & .000 & Sig & Biased against public \\
\hline & Public & 7195 & .6775 & .27316 & & & & & & \\
\hline \multirow[t]{2}{*}{ IT10 } & Private & 6079 & .7409 & .15876 & .02485 & 8.250 & 13272 & .000 & Sig & Biased against public \\
\hline & Public & 7195 & .7160 & 18399 & & & & & & \\
\hline IT11 & Private & 6079 & .7538 & .21897 & .08855 & 18.902 & 13272 & .000 & Sig & Biased against public \\
\hline
\end{tabular}




\begin{tabular}{|c|c|c|c|c|c|c|c|c|c|c|}
\hline \multirow{3}{*}{ IT12 } & Public & 7195 & .6653 & .30479 & & & & & & \\
\hline & Private & 6079 & .6533 & .20332 & .05737 & 13.409 & 13272 & .000 & Sig & Biased against public \\
\hline & Public & 7195 & .5959 & .27632 & & & & & & \\
\hline \multirow[t]{2}{*}{ IT13 } & Private & 6079 & .0806 & .01483 & .01274 & 70.900 & 13272 & .000 & Sig & Biased against public \\
\hline & Public & 7195 & .0678 & .00325 & & & & & & \\
\hline \multirow[t]{2}{*}{ IT14 } & Private & 6079 & .6724 & .23278 & .03701 & 8.227 & 13272 & .000 & Sig & Biased against public \\
\hline & Public & 7195 & .6354 & .27790 & & & & & & \\
\hline \multirow[t]{2}{*}{ IT15 } & Private & 6079 & .6377 & .20878 & .05212 & 12.938 & 13272 & .000 & Sig & Biased against public \\
\hline & Public & 7195 & .5856 & .24862 & & & & & & \\
\hline \multirow[t]{2}{*}{ IT16 } & Private & 6079 & .7707 & .15915 & .01552 & 5.280 & 13272 & .000 & Sig & Biased against public \\
\hline & Public & 7195 & .7551 & .17643 & & & & & & \\
\hline \multirow[t]{2}{*}{ IT17 } & Private & 6079 & .7876 & .21010 & .05255 & 12.363 & 13272 & .000 & Sig & Biased against public \\
\hline & Public & 7195 & .7351 & .26930 & & & & & & \\
\hline \multirow[t]{2}{*}{ IT18 } & Private & 6079 & .5274 & .08339 & .04834 & 29.000 & 13272 & .000 & Sig & Biased against public \\
\hline & Public & 7195 & .4791 & .10496 & & & & & & \\
\hline \multirow[t]{2}{*}{ IT19 } & Private & 6079 & .9142 & .06717 & .03443 & 24.257 & 13272 & .000 & Sig & Biased against public \\
\hline & Public & 7195 & .8798 & .09185 & & & & & & \\
\hline \multirow[t]{2}{*}{ IT20 } & Private & 6079 & .7973 & .14658 & .04477 & 14.675 & 13272 & .000 & Sig & Biased against public \\
\hline & Public & 7195 & .7525 & .19599 & & & & & & \\
\hline \multirow[t]{2}{*}{ IT21 } & Private & 6079 & .4291 & .09815 & .02267 & 11.627 & 13272 & .000 & Sig & Biased against public \\
\hline & Public & 7195 & .4065 & .12239 & & & & & & \\
\hline IT22 & Private & 6079 & .8221 & .18500 & .07248 & 18.471 & 13272 & .000 & Sig & Biased against public \\
\hline & Public & 7195 & .7496 & .25435 & & & & & & \\
\hline IT23 & Private & 6079 & .5847 & .06429 & .08450 & 73.928 & 13272 & .000 & Sig & Biased against public \\
\hline & Public & 7195 & .5002 & .06671 & & & & & & \\
\hline IT24 & Private & 6079 & .7859 & .17454 & .06125 & 18.777 & 13272 & .000 & Sig & Biased against public \\
\hline & Public & 7195 & .7247 & 19737 & & & & & & \\
\hline IT25 & Private & 6079 & .5801 & .00008 & .11514 & 36557.583 & 13272 & .000 & Sig & Biased against public \\
\hline & Public & 7195 & .4649 & .00024 & & & & & & \\
\hline IT26 & Private & 6079 & .8257 & .14033 & .07315 & 23.354 & 13272 & .000 & Sig & Biased against public \\
\hline & Public & 7195 & .7525 & .20737 & & & & & & \\
\hline IT27 & Private & 6079 & .6477 & .16685 & .08293 & 24.778 & 13272 & .000 & Sig & Biased against public \\
\hline & Public & 7195 & .5647 & .21113 & & & & & & \\
\hline IT28 & Private & 6079 & .3751 & .09887 & .02775 & 16.119 & 13272 & .000 & Sig & Biased against public \\
\hline & Public & 7195 & .3473 & .09876 & & & & & & \\
\hline IT29 & Private & 6079 & .4175 & .09370 & .05848 & 32.111 & 13272 & .000 & Sig & Biased against public \\
\hline & Public & 7195 & .3590 & .11288 & & & & & & \\
\hline IT30 & Private & 6079 & .8068 & .13681 & .04644 & 19.286 & 13272 & .000 & Sig & Biased against public \\
\hline & Public & 7195 & .7604 & .13942 & & & & & & \\
\hline IT31 & Private & 6079 & .7190 & .23214 & .06713 & 14.479 & 13272 & .000 & Sig & Biased against public \\
\hline & Public & 7195 & .6519 & 29180 & & & & & & \\
\hline It32 & Private & 6079 & .7578 & 24617 & .06524 & 13.322 & 13272 & .000 & Sig & Biased against public \\
\hline & Public & 7195 & .6926 & .30752 & & & & & & \\
\hline IT33 & Private & 6079 & .7575 & .22468 & .06761 & 15.073 & 13272 & .000 & Sig & Biased against public \\
\hline & Public & 7195 & .6899 & .28225 & & & & & & \\
\hline IT34 & Private & 6079 & .7909 & .21602 & .07938 & 17.470 & 13272 & .000 & Sig & Biased against public \\
\hline & Public & 7195 & .7116 & .29339 & & & & & & \\
\hline IT35 & Private & 6079 & .8199 & .20442 & .09346 & 21.504 & 13272 & .000 & Sig & Biased against public \\
\hline & Public & 7195 & .7265 & .28199 & & & & & & \\
\hline IT36 & Private & 6079 & .6680 & .23256 & .08281 & 18.716 & 13272 & .000 & Sig & Biased against public \\
\hline & Public & 7195 & .5852 & .27078 & & & & & & \\
\hline IT37 & Private & 6079 & .5890 & .24985 & .03677 & 7.995 & 13272 & .000 & Sig & Biased against public \\
\hline & Public & 7195 & .5523 & .27537 & & & & & & \\
\hline IT38 & Private & 6079 & .7239 & .21791 & .00148 & .411 & 13272 & .681 & $\operatorname{sig}$ & Item Impact \\
\hline & Public & 7195 & .7225 & .19740 & & & & & & \\
\hline IT39 & Private & 6079 & .7792 & .19404 & .04915 & 14.972 & 13272 & .000 & Sig & Biased against public \\
\hline & Public & 7195 & .7301 & .18354 & & & & & & \\
\hline IT40 & Private & 6079 & .6566 & 22829 & .06541 & 14.855 & 13272 & .000 & Sig & Biased against public \\
\hline & Public & 7195 & .5912 & 27171 & & & & & & \\
\hline IT41 & Private & 6079 & .6940 & .18108 & -.00295 & -.898 & 13272 & .369 & Sig & Item Impact \\
\hline & Public & 7195 & .6969 & .19449 & & & & & & \\
\hline IT42 & Private & 6079 & .5768 & .23659 & .05419 & 12.524 & 13272 & .000 & Sig & Biased against public \\
\hline & Public & 7195 & .5227 & .25786 & & & & & & \\
\hline IT43 & Private & 6079 & .7409 & .22633 & .02700 & 6.278 & 13272 & .000 & Sig & Biased against public \\
\hline & Public & 7195 & .7139 & .26308 & & & & & & \\
\hline IT44 & Private & 6079 & .6149 & .05819 & .05790 & 55.340 & 13272 & .000 & Sig & Biased against public \\
\hline & Public & 7195 & .5570 & .06160 & & & & & & \\
\hline
\end{tabular}




\begin{tabular}{|c|c|c|c|c|c|c|c|c|c|c|}
\hline \multirow[t]{2}{*}{ IT45 } & Private & 6079 & .7748 & .18063 & .05905 & 16.077 & 13272 & .000 & Sig & Biased against public \\
\hline & Public & 7195 & .7158 & .23334 & & & & & & \\
\hline \multirow[t]{2}{*}{ IT46 } & Private & 6079 & .8452 & .19735 & .07136 & 17.783 & 13272 & .000 & Sig & Biased against public \\
\hline & Public & 7195 & .7739 & .25492 & & & & & & \\
\hline \multirow[t]{2}{*}{ IT47 } & Private & 6079 & .6281 & .14764 & .07009 & 25.759 & 13272 & .000 & Sig & Biased against public \\
\hline & Public & 7195 & .5580 & .16306 & & & & & & \\
\hline \multirow[t]{2}{*}{ IT48 } & Private & 6079 & .7439 & .18626 & .03844 & 11.003 & 13272 & .000 & Sig & Biased against public \\
\hline & Public & 7195 & .7055 & .21188 & & & & & & \\
\hline \multirow[t]{2}{*}{ IT49 } & Private & 6079 & .8255 & .15782 & .04579 & 16.487 & 13272 & .000 & Sig & Biased against public \\
\hline & Public & 7195 & .7797 & .16077 & & & & & & \\
\hline \multirow[t]{2}{*}{ IT50 } & Private & 6079 & .6810 & .15695 & -.00920 & -2.883 & 13272 & .004 & Sig & Biased against public \\
\hline & Public & 7195 & .6902 & .20286 & & & & & & \\
\hline \multirow[t]{2}{*}{ IT51 } & Private & 6079 & .8734 & .10765 & .05308 & 21.994 & 13272 & .000 & Sig & Biased against public \\
\hline & Public & 7195 & .8203 & .16006 & & & & & & \\
\hline \multirow[t]{2}{*}{ IT53 } & Private & 6079 & .8099 & .14225 & .03215 & 12.308 & 13272 & .000 & Sig & Biased against public \\
\hline & Public & 7195 & .7778 & .15619 & & & & & & \\
\hline \multirow[t]{2}{*}{ IT54 } & Private & 6079 & .8135 & .16746 & .03989 & 11.463 & 13272 & .000 & Sig & Biased against public \\
\hline & Public & 7195 & .7736 & .22343 & & & & & & \\
\hline \multirow[t]{2}{*}{ IT55 } & Private & 6079 & .4578 & .08801 & .11674 & 89.107 & 13272 & .000 & Sig & Biased against public \\
\hline & Public & 7195 & .3410 & .06236 & & & & & & \\
\hline \multirow[t]{2}{*}{ IT56 } & Private & 6079 & .7362 & 16779 & .00903 & 3.265 & 13272 & .001 & Sig & Biased against public \\
\hline & Public & 7195 & .7272 & .15086 & & & & & & \\
\hline \multirow[t]{2}{*}{ IT57 } & Private & 6079 & .8012 & .17255 & .05582 & 16.046 & 13272 & .000 & Sig & Biased against public \\
\hline & Public & 7195 & .7454 & .22004 & & & & & & \\
\hline \multirow[t]{2}{*}{ IT58 } & Private & 6079 & .8074 & .15745 & .06526 & 19.632 & 13272 & .000 & Sig & Biased against public \\
\hline & Public & 7195 & .7422 & .21499 & & & & & & \\
\hline \multirow[t]{2}{*}{ IT59 } & Private & 6079 & .6982 & .20021 & .02246 & 5.750 & 13272 & .000 & Sig & Biased against public \\
\hline & Public & 7195 & .6758 & .24264 & & & & & & \\
\hline \multirow[t]{2}{*}{ IT60 } & Private & 6079 & .5670 & .11195 & .06801 & 33.717 & 13272 & .000 & Sig & Biased against public \\
\hline & Public & 7195 & .4990 & .11893 & & & & & & \\
\hline
\end{tabular}

Significant at $\mathrm{p} \leq 0.05$

Table 5 showed the disparity between individual examinee performances on the items, with mean differences in the score at face value among the comparison groups. The table showed that 56 scores $(96 \%$ of items) $(1,2,3,4,6,8,9,10$, $11,12,13,14,15,16,17,18,19,20,21,22,23,24,25,26,27,28,29,30,31,32,33,34,35,36,37,39,40,45,46,47$, $48,49,50,51,52,53,54,55,56,58,59$ and 60 ) were biased against candidates from public schools; while ( $3 \%$ of items) score 38 and 41 showed item impact. This showed that there was a similar bundle of items assembled in the tests that made comparison groups respond equally to these items because item impact reflected the intended purpose of the test. It was apparent that it disadvantaged examinees from public schools on difficult items that required higher calculation skills, perhaps; they were also careless on easier items that require the application of one to more simple basic mathematical operations. Scores of examinees from private schools also indicated that they could answer both difficult and easier items that require higher calculation skills and on item estimates of simple mathematical skills. In comparing scores along with the groups, we might conclude that students from private schools have mastered the subject content well and have confident skills when the test was administered. The results imply that school type is a factor that is irrelevant to the purpose of the test that brought mean differences in score across groups, thereby reflecting differential construct validity and content validity biases of items.

\section{Discussion}

The study under the MTTST method examined the nature of item bias on students' performance in the 2017 NECO SSC dichotomously scored Mathematics test in Nigeria. Findings from the study showed that the 2017 NECO SSC dichotomously scored Mathematics test was essentially unidimensional. The results of factor analysis further revealed that the 2017 NECO SSCE Mathematics items are evidence of unidimensionality as the first eigenvalue factor exceeded that of the second factor with a reasonable distance. This means that the test was accurate in interpreting the behaviour of examinees on the test. Therefore, the assumption holds to a good extent that only one dominant dimension accounted for the variations observed in student's responses to the 2017 NECO Mathematics multiple-choice test items in Nigeria.

These findings support the submission of Ojerinde and Ifewulu (2012) who had earlier investigated test dimensionality using the 2010 UTME Mathematics examination with the outcome that specified that the test fulfilled the assumption of unidimensionality using the MTTST approach. These findings also corroborated the results of the study of Orluwene and Asiegbu (2009) who earlier investigated test dimensionality of Rivers State JSSCE Business Studies using the Item Response Theory approach. Its outcomes indicated that the test items met the assumptions of unidimensionality and local independence. 
Preceding the nature of bias statistically encountered, some preliminary analyses carried out, established a relationship between the test and what was aimed to wholly measure. First, there were indications that the items that reflected DIF in the test represent very hard to very easy items in the subject content of the 2017 NECO Mathematics multiple-choice test blueprint. It implies that not only very difficult items are susceptible to DIF but with easier items as well, despite careful development of items by measurement experts. The study of Madu (2011) provided evidence on gender differences in mathematics multiple-choice test items as it varies according to the content area even when the substance is nearly linked to the course of study. The findings of this study are consistent with the study by Ogbebor and Onuka (2013), Abedalaziz (2011) and Nworgu (2010) who reported the incidence of gender, location and school type DIF in mathematics, Economics and Biology respectively. It means, there were other irrelevant factors such as model misspecification, school location, age, sex, culture, peer group, ethnicity, social values and beliefs that have affected the magnitude to which DIF occurs in these examinations. Findings revealed 0.2 was established as the highest ability estimate of candidates with the same probability of answering the items correctly. It means that successful students with the chance of a maximum score on the test proceeded for further studies, while the chances of unsuccessful examinees continuing their studies are restricted. On another thought, the possibility of examinees to have the same ability estimates on the test by answering the items correctly may also suggest incidences of administrative errors on the part of the examination agency, guessing, easiness of items, test wiseness, ethnicity, test language, item format, cheating, or examination malpractices among the students. If examination agencies do not ensure that test items are free from such factors, decisions made from the scores might overestimate or underestimate students' performance in the process. This implies that any misinterpretation of the examinee latent ability on the scale would negatively affect the validity of the decisions made which may lead to a biased result or incorrect conclusions about individuals from the groups. The findings from the estimation of the probability of correct response of examinees having the same ability estimate based on sex, school location and school type showed the total true score of each candidate on the test. It revealed that male students, students from urban and public schools were disadvantaged on items that required higher calculation skills and perhaps, on easier items that require the application of one to more simple basic mathematical operations. These findings are in disparity with Allahnana et al. (2018) study on gender interest. The researcher observed male students outshined in mathematics achievement tests than their female counterparts. Likewise, the scholar believed that less complex rural lifestyles are more than what is experienced at city centres where cultural diversity affects students' educational achievement. The results of the study of Agbir (2004) were consistent with these findings who discovered that rural area students performed better on realistic abilities in chemistry than their city centres counterparts did. It was clear in this study that candidates from rural areas could perform better than candidates from urban areas, considering the provision of social amenities and opportunities. However, for this construct does not have reliably measure its intended purpose effectively, the factors that brought differences in the performances of students might have affected the reliability of the examination scores. As noted by Geisinger et al (2013), these factors could be indications of time between testing administrations, the similarity of content, changes in subjects over time that is introduced by physical complaints, emotional problems, location of the school, type of school, sex, fatigue, starving, while the test-based agents are poor test instructions, administrative errors, subjective scoring, test wiseness, guessing, cheating, model misspecification, and expectations of subjects regarding different elements of the examination.

\section{Conclusion and Recommendations}

The findings of the study showed that the 2017 NECO Mathematics multiple-choice examination designed for the certification of candidates for higher education exhibits a mean difference in score bias. Based on the findings of this study, the following recommendations were made:

(1) Examination agencies and test developers should be encouraged to consistently have test materials reviewed by experts trained in identifying culturally and linguistically diverse subgroups.

(2) Test experts should incorporate a base for identifying candidates that have the same ability estimate with the highest frequency for bias analysis. This would provide information on the examinee that falls into the same ability group.

(3) Examination agencies and test experts should also subject scores of candidates with the same ability scores by estimating their probability of correct response along with their groups with the model that fitted the data. This would provide information on candidates actual true scores and know whether the items are too easy or too difficult for the student.

(4) Curriculum developers may consider the inclusion of more topics in curriculum design that could measure achievement connected with knowledge and skills students need outside the school. 


\section{References}

Abedalaziz, N. (2011). Detecting dif using item characteristic curve approaches. International Journal of Educational and Psychological Assessment, 8(2), 1-15.

Aborisade, O. J. (2016). Comparative analysis of item bias of the mathematics examination constructed by WAEC and NECO in Nigeria. An unpublished PhD Thesis of the Ekiti State University, Ado- Ekiti, Nigeria.

Adedoyin, O. O. (2010). Using IRT approach to detect gender biased items in public examination: A case study from the Botswana junior certificate examination in mathematics. Educational Research and Reviews, 5(7), $385-399$.

Agbir, E. (2004). The meaning of things. New York: Cambridge University Press.

Ahmad, H., Mokshein, S. E., \& Husin, M. R. (2018). Detecting item bias in an anatomy \& physiology test for nursing students using item response theory. International Journal of Academic Research in Progressive Education and Development, 7(1), 97-109. https://doi.org/10.6007/IJARPED/v7-i1/3904

Allahnana, K. M., Akande, M. T., Vintseh, I. M., Usman, Alaku, E. A., \& Monica, E. A. (2018). Assessment of gender and interest in mathematics achievement in Keffi local government area of Nasarawa State, Nigeria. International Journal of Operational Research in Management, social sciences and Education. IJORMSSE. 5(9), 10-13.

Bandele S. O., \& Aborisade, O. J. (2018). Examining the item bias of mathematics examinations constructed by WAEC and NECO in Nigeria. International Journal of Quantitative and Qualitative Research Methods, 6(2), 1-7.

Federal Republic of Nigeria (2013). National Policy on Education, 2014 Edition, Lagos, NERDC.

Geisinger, K. F., Bracken, B. A., Carlson, J, F., Hansen, J. C., Kuncel, N. R., Reise, S. P., \& Rodriguez, M. C. (2013). APA handbook of testing and assessment in psychology. Volume 3: Testing and assessment in school psychology and education. Washington, DC: APA. https://doi.org/10.1037/14049-000

Jang, E. E., \& Roussos, L. A. (2007). An investigation into the dimensionality of TOEFL using conditional covariance-based nonparametric approach. Journal of Educational Measurement, 44(1), 1-21. https://doi.org/10.1111/j.1745-3984.2007.00024.X

Madu, B. C. (2011). Using transformed item difficulty procedure to assess ggender-relateddifferential item functioning of multiple-choice mathematics items administered in Nigeria Research on Humanities and Social Sciences. ISSN 2224-5766 (Paper) ISSN 2225-0484(Online) 2(6), 41-55.

Nwogu, M. (2010). Achievement in mathematics and science: Do mothers' beliefs matter 12 years later? Journal of Educational Psychology, 96(1), 97-109. https://doi.org/10.1037/0022-0663.96.1.97

Ogbebor, U., \& Onuka, A. (2013). Differential item functioning of economics question papers of national examinations council in Delta State, Nigeria, Nigerian Journal of Educational Research and Education, 12(1), 45-60.

Ojerinde, D., \& Ifewulu, B. C. (2012). Item Unidimensionality Using 2010 Unified Tertiary Matriculation Examination Mathematics Pre-Test. a paper presented at the 2012 International Conference of IAEA. Kazakstan.

Orluwene, G., \& Asiegbu, C. N. (2009). Detecting item bias in Rivers State JSSCE business studies using Item Response Theory (IRT) approach. European Journal of Educational and Development Psychology, 4(1), $42-52$.

Uremu, O., \& Adams, O. (2013). Differential items functioning method as an item bias indication. Journal of Educational Research. 4(4), 367-373.

Zhang, J. (2007). Conditional covariance theory and detect for polytomous items. Psychometrika, 72, 69-91. https://doi.org/10.1007/s11336-004-1257-7

\section{Copyrights}

Copyright for this article is retained by the author(s), with first publication rights granted to the journal.

This is an open-access article distributed under the terms and conditions of the Creative Commons Attribution license which permits unrestricted use, distribution, and reproduction in any medium, provided the original work is properly cited. 